\title{
Mitigation of Amplitude and Phase Distortions by Using Conjugate-NOLM Regenerator
}

\author{
Feng Wen ${ }^{1,2}$, Baojian $W^{2}$, Feng Yang ${ }^{3}$, and Stylianos Sygletos ${ }^{1}$ \\ ${ }^{I}$ Aston Institute of Photonic Technologies, Aston University, B4 7ET Birmingham, UK \\ ${ }^{2}$ Key Lab of Optical Fiber Sensing and Communication Networks, Ministry of Education, University of \\ Electronic Science and Technology of China, 611731 Chengdu, China \\ ${ }^{3}$ Lab of Holographic Optical Sensing, Marolabs Co., Ltd, 610041 Chengdu, China \\ f.wen@aston.ac.uk
}

\begin{abstract}
In this paper we propose a conjugate nonlinear optical loop mirror scheme (Conj-NOLM) by cascading two NOLMs with an intermediate optical phase conjugator stage (OPC). This new configuration utilizes mid-span spectral inversion to cancel out the nonlinear phase distortion that is introduced by the two NOLM units. Moreover, numerical investigation has been carried out for 16-QAM signals demonstrating increased robustness against accumulated amplitude and phase distortions in the transmission links.
\end{abstract}

Keywords: multilevel amplitude and phase noise suppression, conjugate nonlinear optical loop mirror, all-optical regenerator.

\section{INTRODUCTION}

Advanced modulated formats can dramatically increase the system capacity of optical fibre communication networks, but they are vulnerable to nonlinear transmission impairments and amplified spontaneous emission noise (ASE) [1]. All-optical regeneration is a promising technology to deal with these degradations directly in the optical domain and achieve capacities beyond the linear Shannon limit [2]. However, when moving to highconstellation orders simultaneous multilevel suppression of amplitude and phase noise becomes very challenging. Several schemes, such as a modified nonlinear amplifying loop mirror [3] or a regenerative Fourier transform [4], have been proposed by combining phase-sensitive amplifiers (PSAs) and nonlinear interferometer (NI) structures. Among NI configurations, the nonlinear optical loop mirror (NOLM) is a promising candidate for multilevel amplitude regeneration by creating staircase like amplitude transfer function (ATF). However, it also introduces the phase distortion through self-phase modulation (SPM). One can achieve a level of phasepreserving operation in a single NOLM by inserting an attenuator in the high-power arm [5] or by using directional attenuation [6]. However, these techniques are not very power efficient and do not perform well for multi-level signals. In this paper we place an intermediate optical phase conjugator stage (OPC) at a two-NOLM cascaded subsystem, enabling compensation of nonlinear phase distortion through mid-span spectral inversion. Moreover, this scheme is free of the power manipulating devices, which efficiently utilizes the pump power for multilevel amplitude regeneration. By inserting this novel structure at the middle of transmission links the mitigation of total amplitude and phase distortion is successfully achieved.

\section{CONJ-NOLM REGENERATOR}

Figure 1 depicts the proposed conjugate nonlinear optical loop mirror scheme (Conj-NOLM), which includes two NOLM units (NOLM-1 and NOLM-2) and an OPC stage. From the transmission port of the first NOLM (i.e. monitoring point 2) we can get the following output signal [7]:

$$
E_{2}=\exp \left(-\frac{\alpha_{1}}{2} L_{1}\right)\left\{\left(1-\beta_{1}\right) \exp \left[i \gamma_{1}\left(1-\beta_{1}\right) P_{1} L_{1}\right]-\beta_{1} \exp \left(i \gamma_{1} \beta_{1} P_{1} L_{1}\right)\right\} E_{1}
$$

where $E_{1}$ and $P_{1}$ are the input signal and its power; $\alpha_{1}, L_{1}, \gamma_{1}$ are the loss, length and nonlinear coefficient of the highly nonlinear fibre (HNLF-1) in the NOLM-1 unit, respectively; $\beta_{1}$ is the splitting ratio of the optical

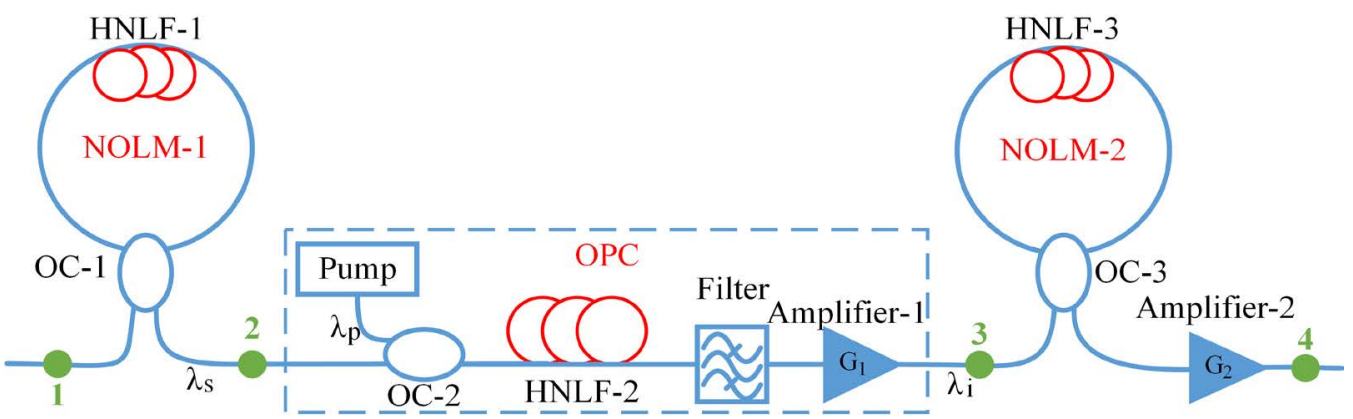

Figure 1. Proposed Conj-NOLM based regenerator subsystem. 
coupler (OC-1). Then, the transmitted signal is coupled into an OPC stage, pumped at $\lambda_{p}$, where four-wave mixing (FWM) enabled wavelength conversion and phase conjugation occur. An optical filter and an amplifier at the OPC output select and boost the generated idler signal at wavelength $\lambda_{i}$ :

$$
E_{3}=\sqrt{G_{1}} \eta E_{2}^{*}
$$

where $G_{1}$ is the gain coefficient of the optical amplifier-1, and $\eta$ is the FWM conversion efficiency. The optical amplifier compensates the power loss of the NOLM-1 unit as well as the effective loss of the phase conjugation, represented by a power compensation parameter $\varepsilon=G_{1} \eta^{2} \exp \left(-\alpha_{1} L_{1}\right)$. The generated idler signal is subsequently launched into a second NOLM unit and at the monitoring point 4 we obtain:

$$
\begin{aligned}
E_{4}=\exp \left(-\frac{\alpha_{1} L_{1}+\alpha_{2} L_{2}}{2}\right) \sqrt{G_{1} G_{2}} \eta E_{1}^{*} & \times\left\{\left(1-\beta_{1}\right) \exp \left[-i \gamma_{1}\left(1-\beta_{1}\right) P_{1} L_{1}\right]-\beta_{1} \exp \left(-i \gamma_{1} \beta_{1} P_{1} L_{1}\right)\right\} \\
& \times\left\{\left(1-\beta_{2}\right) \exp \left[i \gamma_{2}\left(1-\beta_{2}\right) P_{3} L_{2}\right]-\beta_{2} \exp \left(i \gamma_{2} \beta_{2} P_{3} L_{2}\right)\right\}
\end{aligned}
$$

where $\alpha_{2}, L_{2}, \quad \gamma_{2}$ are the loss, length and nonlinear coefficient of the highly nonlinear fibre (HNLF-3) in the NOLM-2 unit, respectively; $G_{2}$ is the gain coefficient of the optical amplifier-2, placed at the transmission port of the NOLM-2 unit; $\beta_{2}$ is the splitting ratio of the optical coupler (OC-3); the optical power of the boosted idler at the monitoring point 3 is $P_{3}=G_{1} \eta^{2} \exp \left(-\alpha_{1} L_{1}\right)\left[1-2 \beta_{1}\left(1-\beta_{1}\right)\left(1-\cos \phi_{1}\right)\right] P_{1}$, where the nonlinear phase shift is $\phi_{1}=\gamma_{1}\left(2 \beta_{1}-1\right) P_{1} L_{1}$.

\section{GAIN OPTIMIZATION}

Equation (1) governs the output of a single NOLM unit, which it does not only provide a multilevel regenerative response in the amplitude, but also a self-phase modulation through the $\exp \left(i \gamma_{1} \beta_{1} P_{1} L_{1}\right)$ term, which can distort severely the incoming signal. The intermediate OPC stage can be used to cancel out the generated phase distortion of the two interferometers in the regenerator setup. Moreover, when placing the proposed Conj-NOLM regenerator at the middle of the link, deterministic nonlinear phase distortions that have been symmetrically generated along the transmission link can be also cancelled through mid-span spectral inversion. To this end, power symmetry is crucial both at subsystem and transmission link level. However, considering the nonlinear response of each NOLM interferometer joint gain optimization of the two optical amplifiers in the subsystem will be required to successfully combine the OPC and the two NOLM units, enabling mitigation of the total amplitude and phase distortion in the transmission link.

We firstly focus on cancelling the nonlinear phase distortion of the two NOLM units in the regenerator subsystem. Power symmetry requires the instantaneous signal levels at the input of NOLM-1 and NOLM-2 to be identical, i.e. $P_{3}=P_{1}$. In this ideal case, the phase distortion that is introduced by the first NOLM is completely cancelled by the combination of the OPC stage and the subsequent identical NOLM. However, this strict condition also requires an instantaneous variation gain related to the optical power of each symbol $G_{1}=1 / \eta^{2} \exp \left(-\alpha_{1} L_{1}\right)\left[1-2 \beta_{1}\left(1-\beta_{1}\right)\left(1-\cos \phi_{1}\right)\right]$, see $\phi_{1}$ in the cosine function, which is experimentally impossible by Erbium-doped fibre amplifiers (EDFAs). On the contrary, what can be achieved with a fixed gain amplifier is a "sub-symmetry" condition where the first NOLM is optimized to have an almost linear amplitude response and to introduce at the same time a phase pre-distortion, which can be used to minimize the nonlinear phase shift of
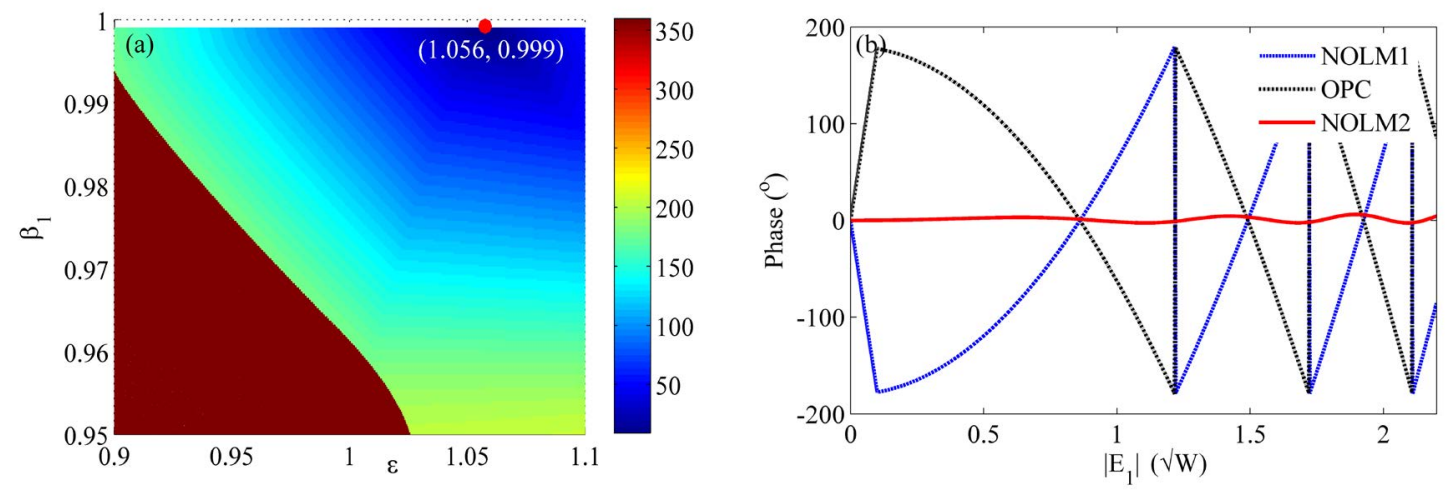

Figure 2:(a) Max phase change with the splitting ratio $\beta_{1}$ and the power compensation parameter $\varepsilon$; (b) phase evolution at the output of the NOLM-1, the OPC and the NOLM-2.

the second NOLM unit. Therefore, the optimization focuses on the splitting ratio $\beta_{1}$ of the NOLM-1 unit and the power compensation parameter $\varepsilon$ defined in the OPC unit. The details of the optimization procedure are as follows: we firstly increase the input amplitude $\left|E_{1}\right|$ from 0 to $2.2 \sqrt{\mathrm{W}}$, which can support a three-level 
amplitude regeneration, e.g. 16-QAM signals. Then we calculate the maximal phase change $\Delta \phi$ (in degree) of the output signal $E_{4}$ of the whole regenerator subsystem, achieved in such amplitude range. Subsequently we plot the phase change $\Delta \phi$ with the function of the splitting ratio $\beta_{1}$ and the power compensation parameter $\varepsilon$, see Fig. 2(a). Other parameters were used in the simulation similar to those reported in the experiment of [7]: the nonlinear coefficient $\gamma$ was $7 \mathrm{~W}^{-1} / \mathrm{km}$, the total loss was $12 \mathrm{~dB}$ and the fibre length $L$ was $606 \mathrm{~m}$. The splitting ratio $\beta_{2}$ of the second NOLM was chosen to be 0.95 which enabled an effective amplitude noise suppression. From Fig. 2(a) we locate the minimal value of $\Delta \phi=8.8^{\circ}$ with the optimal parameters of $\beta_{1}=0.999$ and $\varepsilon=1.056$. As we can see, the first NOLN becomes highly asymmetrical, which gives a less effect on the signal amplitude, but it can introduce an essential phase pre-distortion. Although the coupling ratio of the second NOLM is different from the first NOLM we can still minimize the total phase change on the output signal. Figure 2(b) plots the phase evolution at the different stages of the optimized Conj-NOLM regenerator. A large phase distortion is clearly observed after the first NOLM. By optimizing the gain strength and the coupling ratio, the phase change of the output signal has been significantly reduced to $8.8^{\circ}$. This residual distortion comes from the power asymmetry within the Conj-NOLM regenerator.

Then we calculate the gain coefficient $G_{2}$ of the optical amplifier-2 when the proposed regenerator is inserted into the middle of the link. Power symmetry requires the signal levels at the two fibre links to be identical, which means that the optical amplifier-2 compensates the power loss from the Conj-NOLM regenerator as well as the propagation loss of the fibre link. Here we only compare the average optical powers at the output (monitoring point 4) and the input (monitoring point 1), leading to $G_{2}=17.6$ for the loss from the regenerator subsystem.

\section{DISTORTION MITIGATION}

In this section we examined the performance of the proposed regenerator in a cascaded transmission scenario. We considered a hypothetical transmission system of a signal located in the zero-dispersion wavelength passing through $\mathrm{N}$ cascaded regenerative transmission sections. The Conj-NOLM regenerator was placed in the middle of each of those section, whereas fibre spans before and after the regenerator introduced nonlinear phase distortion of $\varphi_{N L}=\gamma L_{e f f} P_{s}=28 P_{s}$ in which $P_{s}$ was the instantaneous power of each symbol. ASE noise was added separately. The system performance was compared with and without the existence of the regenerator setup, see Fig. 3.

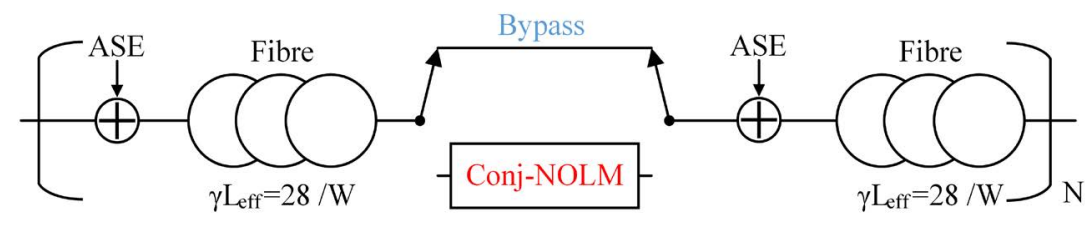

Figure 3. Transmission evaluation with/without the Conj-NOLM regenerator.

We considered at the transmitter a 16-QAM signal with constellation adapted to the nonlinear response of the proposed Conj-NOLM regenerator subsystem, see white constellation in the insert of Fig. 4(a), similarly to what we did in [8]. Then we launched the signal into the transmission system, that comprised of $N=5$ cascaded sections, and we evaluated the corresponding performance through direct error counting. White Gaussian noise was added linearly to the 16-QAM signal so that the signal-to-noise ratio (SNR) was kept fixed at $25 \mathrm{~dB}$. It is obvious that at higher launched powers the transmitted signal experiences a stronger nonlinear phase distortion. Figure 4(a) depicts the calculated bit-error rates (BERs) at the receiver as a function of the launched optical power. In the bypass, i.e. un-regenerated case, a BER of $1.9 \times 10^{-2}$, which corresponds to the FEC limit [9], is calculated for a launched power of $2.4 \mathrm{dBm}$. Including the Conj-NOLM regenerator in the link allows an $8.5 \mathrm{~dB}$ increase in the launched power, which suggests a significant improvement in terms of total transmission reach. In the insert of Fig. 4 we have plotted the signal constellations when the launched power was $5 \mathrm{dBm}$, with and without the use of the Conj-NOLM regenerator in the link. We can see that the use of the regenerator enables a significant reduction of both amplitude and phase distortions. A clearer evidence of the achieved degradation reduction is depicted in the amplitude and phase histograms of Figs. 4(b) and (c), respectively. The three discrete amplitude levels of the signal and the corresponding noise suppression are clearly observed in Fig. 4(b) when we include the optical regenerator in the link. In the phase histograms of Fig. 4(c) we can also clearly identify the twelve phase states of the 16-QAM constellation, since the presence of the symmetrically located OPC in the link prevents the accumulation of the nonlinear phase distortion in the transmission system. The results confirm that the proposed regenerator can successfully combine the operations of the OPC and the NOLM units for longhaul transmission improvement. A more thoughtful investigation by taking into account also the impact of chromatic dispersion can be carried out as a next step, to get a more accurate estimation of the achieved performance improvement. 

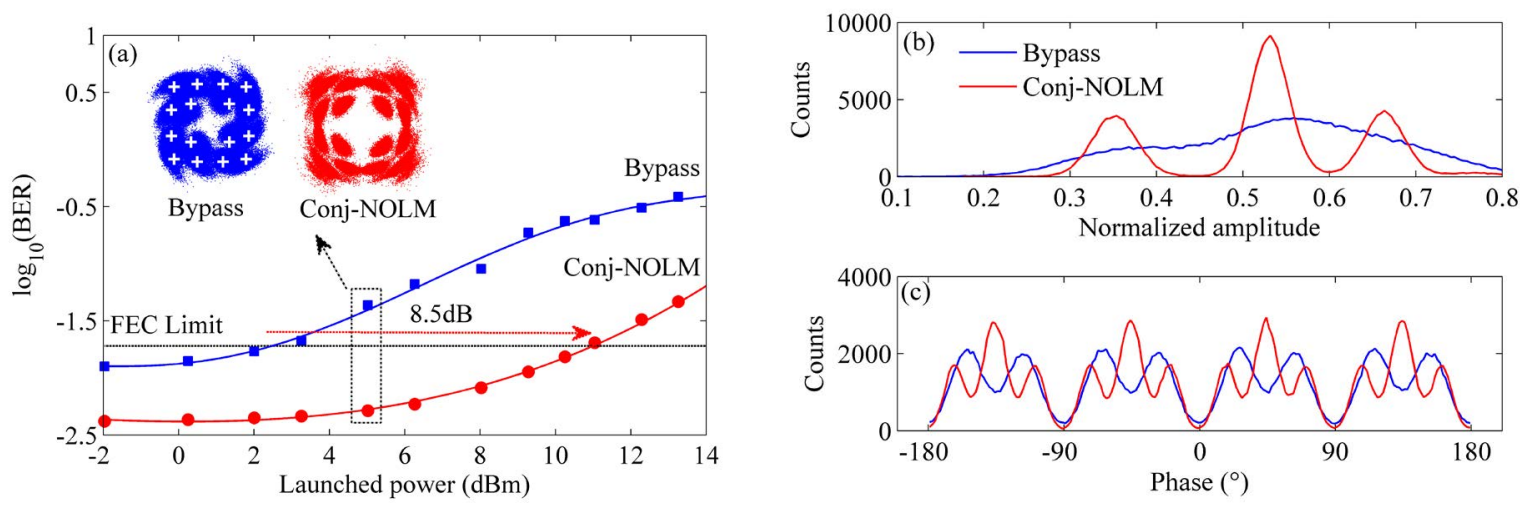

Figure 4. (a) BER results vs. launched optical powers with/without Conj-NOLM regenerator, histogram results of (b) amplitude and (c) phase between bypass and Conj-NOLM cases at the launched power of $5 \mathrm{dBm}$.

\section{CONCLUSIONS}

We proposed a Conj-NOLM regenerator subsystem to achieve a phase-preserving operation by cascading two NOLM units with an intermediate OPC stage. By optimizing the coupling ratio of the first NOLM and the gain coefficient of the optical amplifier, the NOLM-induced phase distortion was significantly reduced to $8.8^{\circ}$. Moreover, by placing the Conj-NOLM regenerator at the middle of transmission links, we simultaneously reduced the signal degradations from both the ASE noise and the nonlinear phase distortions generated along the links.

\section{ACKNOWLEDGEMENTS}

The authors would like to thank Mohammad A. Z. Al-Khateeb, Mingming Tan and Lukasz Krzczanowicz for the valuable discussions. This work has been supported by the EPSRC project UNLOC (EP/J017582/1), the National Natural Science Foundation of China (61505021, 61671108), the Marie Skłodowska-Curie Action (701770-INNOVATION), the General Project of Sichuan Provincial Education Department (18ZB0235) and the 111 Project (B14039).

\section{REFERENCES}

[1] A. Carena et al.: Maximum reach versus transmission capacity for Terabit superchannels based on 27.75GBaud PM-QPSK, PM-8QAM, or PM-16QAM, IEEE Photon. Technol. Lett., vol. 22, pp. 829-831, Mar. 2010.

[2] M. A. Sorokina and S. K. Turitsyn: Regeneration limit of classical Shannon capacity, Nat. Commun., vol. 5, pp. 3861, May 2014.

[3] T. Roethlingshoefer et al:: Multilevel amplitude and phase regeneration in a nonlinear amplifying loop mirror with a phase-sensitive amplifier, in Proc. ECOC, Amsterdam, Netherlands, Sep. 2012, paper Tu.1.A.3.

[4] M. Sorokina et al:: Regenerative Fourier transformation for dual-quadrature regeneration of multilevel rectangular QAM, Opt. Lett., vol. 40, pp. 3117-3120, Jul. 2015.

[5] T. Roethlingshoefer et al.: All-optical phase-preserving multilevel amplitude regeneration, Opt. Express, vol. 22, pp. 27077-27085, Oct. 2014.

[6] Arne G. Striegler et al.: NOLM-based RZ-DPSK signal regeneration, IEEE Photon. Technol. Lett., vol. 17, pp. 639-641, Mar. 2005.

[7] F. Wen et al:: Multilevel power transfer function characterization of nonlinear optical loop mirror, in Proc. ICTON, Girona, Spain, Jul. 2017, paper We.D5.3.

[8] F. Wen et al.: Phase-preserving multilevel amplitude regeneration in conjugate nonlinear-optical loop mirror pair, in Proc. CLEO, San Jose, America, May 2018, paper SM4K.6.

[9] G. Tzimpragos et al.: A survey on FEC codes for 100G and beyond optical networks, Commun. Surveys Tutorials, vol. 18, pp. 209-221, Oct. 2016. 